\title{
Carbazochrome Carbon Nanotube as Drug Delivery Nanocarrier for Anti-bleeding Drug: Quantum Chemical Study
}

\section{Hakan. S. Sayiner}

Adiyaman University Faculty of Medicine: Adiyaman Universitesi Tip Fakultesi

Fatma Kandemirli ( $\square$ fkandemirli@yahoo.com )

Kastamonu Üniversitesi: Kastamonu Universitesi https://orcid.org/0000-0001-6097-2184

Serap Senturk Dalgic

Trakya University: Trakya Universitesi

Majid Monajjemi

Kastamonu Üniversitesi: Kastamonu Universitesi

Fatemeh Mollaamin

Kastamonu Üniversitesi: Kastamonu Universitesi

\section{Research Article}

Keywords: CNT, Carbazochrome, Drug delivery, ELF, QTAIM

Posted Date: September 15th, 2021

DOI: https://doi.org/10.21203/rs.3.rs-857218/v1

License: (c) (1) This work is licensed under a Creative Commons Attribution 4.0 International License. Read Full License

Version of Record: A version of this preprint was published at Journal of Molecular Modeling on December 20th, 2021. See the published version at https://doi.org/10.1007/s00894-021-04948-1. 


\title{
Carbazochrome carbon nanotube as drug delivery nanocarrier for anti- bleeding drug: Quantum Chemical Study
}

\author{
Hakan S. Sayiner ${ }^{1}$, Fatma Kandemirli ${ }^{* 2}$, Serap Senturk Dalgic ${ }^{3}$, Majid Monajjemi² ${ }^{2}$ Fatemeh Mollaamin ${ }^{2}$ \\ ${ }^{1}$ Department of Clinical Infectious Diseases and Medical Microbiology, Faculty of Medicine, Adiyaman \\ University, Training and Research Hospital, Adlyaman, Turkey, \\ ${ }^{2}$ Biomedical Engineering Department, Faculty of Engineering \& Architecture, Kastamonu University, \\ Kastamonu, Turkey fkandemirli@yahoo.com ORCID: 0000-0001-6097-2184
}

${ }^{3}$ Department of Physics, Trakya University, 22030 Edirne, Turkey

\begin{abstract}
The interaction between drugs and single-walled carbon nanotubes is proving to be of fundamental interest for drug system of delivery and nano-bio-sensing. In this study, the interaction of pristine CNT with carbazochrome, an anti-hemorrhagic or hemostatic agent was investigated with M06-2X functional and 6-31G $\mathrm{G}^{*}$ and $6-31 \mathrm{G}^{* *}$ basis sets. All probable positions of related adsorption for these kind drugs were thought-out to find out which one is energetically suitable. Based on the achieved data, the stronger interactions appeared the oxygen atom of $\mathrm{C}=\mathrm{O}$ group and nitrogen atom of imine groups. The topology analysis of QTAIM (quantum theory of atoms in a molecule) method was accomplished to understand the properties of interactions between the CNT and Carbazochrome. Frontier molecular orbital energies of all systems, global index including stiffness, softness, chemical Gibbs energies and electrophilicity parameters, as well as some other important physical data such as dipole moment, polarizability, anisotropy polarisibility and hyperpolaribility were calculated, evaluated and then compared together. The essence of the formed bonding model progress along the reaction roots were further validated using electron localization function (ELF) calculations. In addition the total, partial, and overlapping population densities of states were calculated for any further discussion. The highest values of adsorption energies were determined in the range of 18.24) up to (22.12) $\mathrm{kcal} \mathrm{mol}^{-1}$ for these kind systems. The acceptable recovery time of $849 \mathrm{~s}$ was obtained for the desorption of carbazochrome from the CNT surface under UV-Light. The final results exhibit that carbazochrome can serve as a promising carrier and also as sensitive sensors in any kind of practical application.
\end{abstract}

Keyword: CNT; Carbazochrome; Drug delivery; ELF; QTAIM

\section{Introduction}

Spontaneous bleeding control is a concern for surgeons, anesthetists, hematologists and the patient. The spontaneous cessation of bleeding caused by rupture of blood vessels is called hemostasis. Extravasation would be stopped if any holes can be blocked in the system or if the pressure becomes lower than the pressure outside the bleeding vessel [1]. Carbazochrome, an antihemorrhagic or hemostatic agent, causes blocking of blood flow by forming a platelet plug of platelets in the blood. Due to the unique property of the molecule, the drug can be used to prevent excessive blood flow during surgical operations and to treat hemorrhoids [2]. IUPAC name of carbazochrome is 3,6-dihydroxy-1-methyl-2,3-dihydroindol-5-yl)iminourea. They analyzed thermodynamically stable crystal packs of carbazochrome conformers from the stability and properties of the global minimum carbazochrome produced from lattice energy optimisation applying in the repulsion/attraction or dispersion potential field, hydrogen bond analysis and second derivative properties [3].

The physical chemistry data and also pharmacological properties of the bioactive product can be affected through the physiological situation [4]. A drug delivery system (DDS) can be used to increase the effectiveness of drugs and prevent them from being deactivated until to the target areas [5]. Various categories of carriers have been studied, containing lipids, PEGs, polypeptides [6, 7]. Among the many delivery systems, (n, n) single- 
walled carbon nanotubes, composed of armchair atom arranging in a tubular form, appear to represent a promising option [8]. Findings regarding the SWNT-(streptavidin protein) of conjugates to human promyelocytic leukemia (HL60) and human T cells, have been reported by this work [9].

They examined the $(6,6)$ SWCNTs-fluorouracil and p-Sulphonato-calix aren [4] -fluorouracil composite with DFT method in view point of drug delivery [10]. They investigated the potentiality of SWCNTs as a carrier for droksidopa by quantum mechanical calculation and also by molecular dynamics simulation in both gas and water phases [11]. With the bibliography searching, we know, there is no earlier report about using CNT as a nanocarrier for Carbazochrome drug. Therefore, the goal of this study is to investigate the quantum-chemical aspects of the interaction of this drug with SWCNTs.

\section{Computational details}

Through a theoretical quantum calculation, equilibrium geometries optimization, total energies and electronic densities were accomplished in the DFT framework with M062X functional and 6-31G (d) basis set were made using the Gaussian 09 program package [12].

The adsorption behavior of carbazochrome on a zigzag (4,0)SWCNTs (40atom). was investigated. The adsorption energies of carbazochrome on SWCNTs via the different active sites were calculated using the following relationship:

$$
E=E_{\text {complex }}-\left(E_{\text {drug }}+E_{C N T}\right)
$$

Where $E_{\text {drug: }}$ is the energy of the free drug, $E_{\mathrm{CNT}}$ : is the energy of the free carbon nanotube. The binding energy has been corrected using the basis set superposition error (BSSE).

Also, the relaxed CNT-drug system strength is defined as the difference between the energy of interaction $\left(\mathrm{E}_{\text {int }}\right)$ with the energy of the complex and the drug molecule bonded to the SWCNTs in the optimized complexes

$$
E_{\text {int }}=E_{\text {complex }}-\left(E_{\text {drug in complex }}+E_{C N T \text { in complex }}\right)
$$

If $E_{\text {int }}$ is negative, it indicates pull between drug and CNT, if $E_{\text {inter }}$ is positive, it indicates push between drug and CNT.

$\mathrm{E}_{\mathrm{def}}$, can be denoted as the used energy for changing the molecule from its ideal configuration to the relaxed molecule-surface system.

$$
E_{\text {def }}=E_{\text {def-drug }}+E_{\text {def-CNT }}
$$

where $E_{d e f-C N T}$ is the energy of the deformed CNT surface after drug adsorption. $E_{\text {def-drug }}$ is the energy of the drug form molecule with deformed geometry after its adsorption on CNT. The counter-poise (CP) method of Boys and Bernardi was used to correct the $E_{a d s}$ and $E_{\text {int }}$ based on BSSE [13].

The nature of intermolecular energies between drug and CNT surfaces is investigated by using Bader's QTAIM theory using MULTIWFN software [14]. QTAIM theory is used to examine the bond critical points (BCP) between two adjacent atoms: their electron densities and Laplacians [15].

Parameters related to HOMO and LUMO energies such as stiffness, softness, chemical Gibbs energies, electronegativity, chemical potential and electrophility index, nucleofugality, electrofugality, maximum electron flow were calculated using the following equations.

$$
\begin{aligned}
& \Delta E=E_{\text {LUMO- }} E_{\text {HOMO }} \\
& \mu=-\chi \cong\left(\frac{E_{H O M O}+E_{L U M O}}{2}\right)
\end{aligned}
$$




$$
\begin{aligned}
& \eta=\frac{E_{L U M O}-E_{\text {HOMO }}}{2} \\
& \sigma=\frac{\eta}{2} \\
& \omega=\frac{\mu^{2}}{2 \eta} \\
& \Delta E_{n}= \pm \frac{(\mu+\eta)^{2}}{2 \eta} \\
& \Delta E_{e}=\frac{(\mu-\eta)^{2}}{2 \eta} \\
& \Delta N_{\text {ideal }}=-\frac{\mu}{\eta}
\end{aligned}
$$

\section{Current electron density, ELF and LOL functions:}

The current densities can be formulated as following:

$$
\rho(r)=\eta_{i}\left|\varphi_{i}(r)\right|^{2}=\sum_{i} \eta_{i}\left|\sum_{l} C_{l, i} \chi_{i}(r)\right|^{2}
$$

That $\chi$ and $\eta_{i}, \mathrm{C}$ are the basis function for orbitals, occupation number. And a coefficient matrix. Bader [16,17] illustrated, regions of large electron localization have a wide value of Fermi-hole. Becke and coworkers [18] illustrated that spherically average like-spin pair has suitable correlation with the Fermi hole. Consequently, they introduced a new function as "electrons localization functions"(19, 20).

$$
(\mathrm{ELF}) . \operatorname{ELF}(\mathrm{r})=\frac{1}{1+\left[D(r) / D_{0}(r)\right]^{2}}
$$

where

$$
\mathrm{D}(\mathrm{r})=\frac{1}{2} \sum_{i} \eta_{i}\left|\nabla \varphi_{i}\right|^{2}-\frac{1}{8}\left[\frac{\left.|| \nabla \rho_{\alpha}\right|^{2}}{\rho_{\alpha}(r)}+\frac{\left.|| \nabla \rho_{\beta}\right|^{2}}{\beta(r)}\right]
$$

and

$$
D_{0(r)}=\frac{3}{10}\left(6 \pi^{2}\right)^{\frac{2}{3}}\left[\rho_{\alpha}(r)^{\frac{5}{3}}+\rho_{\beta}(r)^{\frac{5}{3}}\right]
$$

for closed-shell systems, since $\rho_{\alpha}(r)=\rho_{\beta}(r)=\frac{1}{2} \rho, D$ and $D_{0}$ terms can be simplified as

$$
\mathrm{D}(\mathrm{r})=\frac{1}{2} \sum_{i} \eta_{i}\left|\nabla \varphi_{i}\right|^{2}-\frac{1}{8}\left[\frac{|\nabla \rho|^{2}}{\rho(r)}\right]
$$

and

$$
D_{0(r)}=\frac{3}{10}\left(3 \pi^{2}\right)^{\frac{2}{3}} \rho(r)^{\frac{5}{3}}
$$

Savin et al. [21], showed, $D(\mathbf{r})$ parameter as the excess kinetic energies due to the Pauli repulsion, while $D_{0}(\mathbf{r})$ is known as Thomas-Fermi kinetic term. Here the ELF can be interpreted in view point of kinetic energies among Kohn-Sham DFT's wave-functions. And it would be in the range of [0,1], which means for a large ELF value electrons are strongly localized. Although ELF can be used for the wide varieties of molecules; Localized orbital locator (LOL) is the other important function that has been investigated by Becke and coworkers instead of ELF for some special molecules [22].

$$
\operatorname{LOL}(r)=\frac{\tau(r)}{1+\tau(r)}, \text { where }(r)=\frac{D_{0}(r)}{\frac{1}{2} \sum_{i} \eta_{i}\left|\nabla \varphi_{i}\right|^{2}}
$$

$D_{0}(r)$ is used synonymously with ELF for spin polarized and closed shell systems.

Jacobsen showed that the kinetic energies of LOL can be interpreted much better than ELF, since LOL provides a more precise and clear image than ELF. LOL can also be interpreted in terms of localized orbitals. LOL's range of values is between zero and one, as with ELF. [0, 1]. 


\section{Result and Discussion}

Various computational tools can be used, such as DFT, CarParrinello MD simulations, and hybrid QM / MM approximations for any further calculations. Quantum molecular calculations, has been provided, recently as a relatively consistent phenomenon of the interaction forces and geometries about the structure, charge distribution, and energy of these kind interacting molecules. Various interaction orientations considered for the interacting drug molecule (N1, O2, N3, N4, which are the semicarbazide group, and the interactions between the O5, N6 and 07 atoms belonging to the indole group) and the CNT were calculated with DFT method in level M062X/6-31G (d) and given in Fig. 1 that exhibits the optimized compound carbazozchrome, a zigzag CNT $(4,0)(40 \mathrm{atom})$, carbazochrome-CNT(4,0) - complexes (complex1-complex6). From Fig. 1 it can be concluded that Carbazochrome has various sites for interaction.
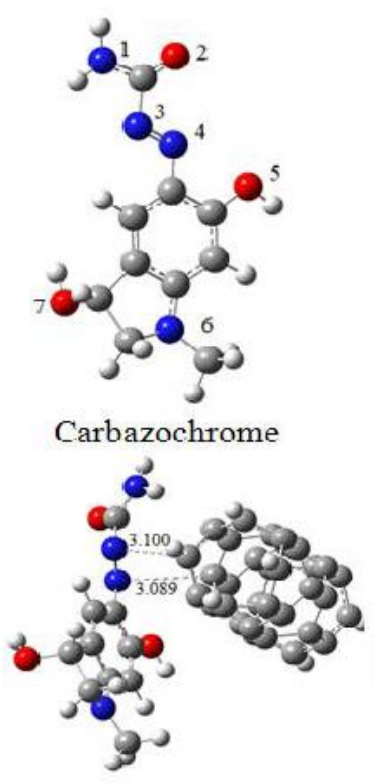

3

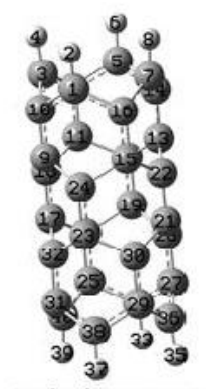
pristine CNT

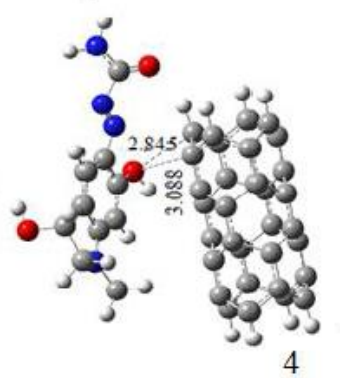

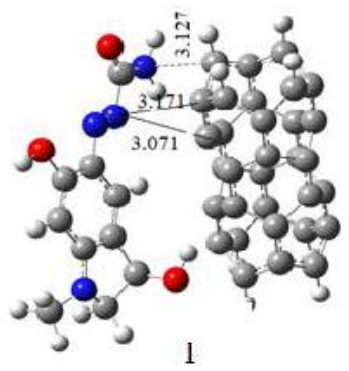

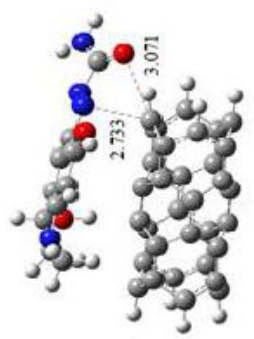

5

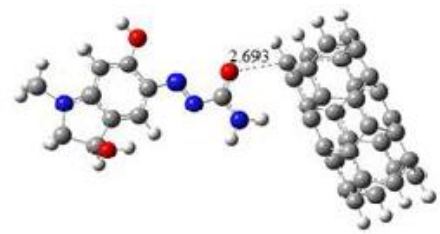

2

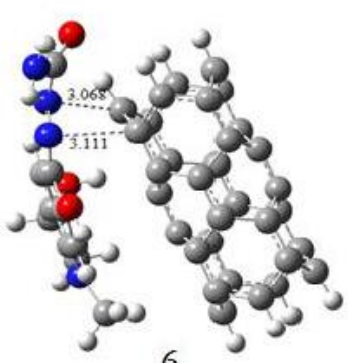

Fig. 1 The optimized compound carbazozchrome, nanotube $(4 \mathrm{x} 0)$, nanotube $(4 \mathrm{x} 0)$-carbazochrome complexes (complex1-complex6) calculated by M062X/6-31G (d) level

The calculated equilibrium binding distance between carbazochrome and CNT for the complex1-complex6 are given in Table 1 and the calculated interaction, adsorption, and deformation energies of complex1-complex6 is given in Table 2. The calculated equilibrium binding distance for N1-C38, N1-C31, N3-C32 and O6-C2 at complex1, are 3.127, 3.171, 3.071, 3.055 $\AA$, respectively, and adsorption energy is $-14.96 \mathrm{kcal} \mathrm{mol}^{-1}$. The energy of adsorption and equilibrium binding distance between $\mathrm{O} 2$ and $\mathrm{C}$ atom belonging to $\mathrm{CNT}$ for the complex 2 are determined to be respectively $7.13 \mathrm{kcal} \mathrm{mol}^{-1}$ and $2.693 \AA$; for complex3, C23-N4, C38-N3 distances are 3.089 and $3.100 \AA$. and calculated adsorption energy is $-8.14 \mathrm{kcal} \mathrm{mol}^{-1}$ for complex 2. Calculated adsorption energies for complex4-complex6 are -18.24, $-22.25,-22.12 \mathrm{kcal} \mathrm{mol}^{-1}$, respectively. Our results suggest that considering only three positions is more energetically important; via $-\mathrm{O}-\mathrm{H}$ attached to the phenyl ring (complex4), via the $\mathrm{O}$ atom of the carbonaceous group and a nitrogen atom of the imine group (complex5), and two nitrogen atoms of imine group (complex6). It was found that the complex5 gives the strongest adsorption energy in the other configurations. 
Table 1 The calculated equilibrium binding distance between carbazochrome and CNT for the complex1complex6

\begin{tabular}{|c|c|c|c|c|c|c|c|c|c|}
\hline & \multirow[t]{2}{*}{ Atom } & \multirow{2}{*}{$\begin{array}{l}\text { Bond } \\
\text { Length }\end{array}$} & \multirow[t]{2}{*}{ Atom } & \multicolumn{2}{|c|}{ Mulliken charges } & \multirow[b]{2}{*}{ Atom } & \multicolumn{2}{|c|}{ Mulliken charges } & \multirow{2}{*}{$\begin{array}{l}\text { Charge } \\
\text { Flow }\end{array}$} \\
\hline & & & & $\mathrm{CNT}$ & Comp & & Drug & Comp & \\
\hline \multirow[t]{4}{*}{1} & C38-N1 & 3.127 & C38 & -0.205 & -0.196 & N1 & -0.815 & -0.796 & \\
\hline & N3-C31 & 3.171 & $\mathrm{C} 31$ & 0.045 & 0.0558 & N3 & -0.339 & -0.316 & \\
\hline & C32-N3 & 3.071 & $\mathrm{C} 32$ & -0.014 & 0.0092 & N3 & -0.339 & -0.316 & -0.02296 \\
\hline & $\mathrm{C} 3-\mathrm{O} 7$ & 3.055 & $\mathrm{C} 3$ & -0.205 & -0.169 & $\mathrm{O} 7$ & -0.645 & -0.666 & \\
\hline \multirow[t]{3}{*}{2} & $\mathrm{C} 38-\mathrm{O} 2$ & 2.693 & C38 & -0.205 & -0.153 & $\mathrm{O} 2$ & -0.482 & -0.507 & \\
\hline & O2-H37 & 2.556 & H37 & 0.189 & 0.215 & $\mathrm{H}$ & 0.392 & 0.368 & 0.004935 \\
\hline & $\mathrm{H}-\mathrm{C} 23$ & 2.277 & $\mathrm{C} 23$ & -0.014 & -0.104 & & & & \\
\hline \multirow[t]{2}{*}{3} & C23-N4 & 3.089 & $\mathrm{C} 23$ & -0.014 & 0.008 & N4 & -0.227 & -0.216 & 0.007103 \\
\hline & C38-N3 & 3.100 & C38 & -0.205 & -0.171 & N3 & -0.339 & -0.315 & \\
\hline \multirow[t]{2}{*}{4} & C38-O5 & 2.845 & C38 & -0.205 & -0.160 & O5 & -0.632 & -0.653 & 0.029106 \\
\hline & C31-O5 & 3.088 & $\mathrm{C} 31$ & 0.045 & 0.030 & & & & \\
\hline \multirow[t]{2}{*}{5} & N4-C38 & 2.733 & C38 & -0.205 & -0.178 & N4 & -0.216 & -0.250 & 0.002562 \\
\hline & $\mathrm{O} 2-\mathrm{C} 38$ & 3.071 & & & & $\mathrm{O} 2$ & -0.482 & -0.525 & \\
\hline \multirow[t]{2}{*}{6} & N4-C31 & 3.111 & C31 & 0.045 & 0.047 & N4 & -0.227 & -0.279 & 0.002217 \\
\hline & N3-C38 & 3.068 & C38 & -0.205 & -0.062 & N3 & -0.339 & -0.297 & \\
\hline
\end{tabular}

Table 2 The calculated interaction, adsorption, and deformation energies of Carbazochrome, CNT, and complex1-complex6 $\left(\mathrm{kcal} \mathrm{mol}^{-1}\right)$

\begin{tabular}{|c|c|c|c|c|c|}
\hline Complex & $\mathrm{E}_{\text {ads/inter/def }}$ & SEZPE & SETE & SETEn & SETFE \\
\hline \multirow[t]{3}{*}{1} & $E_{a d s}$ & -14.96 & -14.05 & -14.65 & -1.72 \\
\hline & $E_{\text {inter }}$ & -16.45 & -15.53 & -16.13 & -2.59 \\
\hline & $E_{d e f}$ & 1.49 & 1.48 & 1.48 & 0.87 \\
\hline \multirow[t]{3}{*}{2} & $E_{a d s}$ & -7.13 & -5.91 & -6.51 & 3.54 \\
\hline & $E_{\text {inter }}$ & -7.55 & -6.26 & -6.85 & 3.95 \\
\hline & $E_{\text {def }}$ & 0.42 & 0.34 & 0.34 & -0.41 \\
\hline \multirow[t]{3}{*}{3} & $E_{a d s}$ & -8.14 & -6.99 & -7.58 & 3.81 \\
\hline & $E_{\text {inter }}$ & -10.29 & -8.96 & -9.56 & 2.33 \\
\hline & $E_{d e f}$ & 2.15 & 1.97 & 1.97 & 1.48 \\
\hline \multirow[t]{3}{*}{4} & $E_{a d s}$ & -18.24 & -17.11 & -17.71 & -5.64 \\
\hline & $E_{\text {inter }}$ & -21.28 & -20.21 & -20.81 & -7.99 \\
\hline & $E_{d e f}$ & 3.04 & 3.10 & 3.10 & 2.35 \\
\hline \multirow[t]{3}{*}{5} & $E_{a d s}$ & -22.25 & -21.50 & -22.09 & -8.25 \\
\hline & $E_{\text {inter }}$ & -24.23 & -23.33 & -23.93 & -9.84 \\
\hline & $E_{\text {def }}$ & 1.98 & 1.84 & 1.84 & 1.59 \\
\hline \multirow[t]{3}{*}{6} & $E_{a d s}$ & -22.12 & -21.50 & -22.09 & -8.11 \\
\hline & $E_{\text {inter }}$ & -24.11 & -22.76 & -23.35 & -10.13 \\
\hline & $E_{d e f}$ & 1.99 & 1.26 & 1.26 & 2.01 \\
\hline
\end{tabular}

SEZPE:Sum of electronic and zero-point Energies SETE:Sum of electronic and thermal Energies

SETEn: Sum of electronic and thermal Enthalpies SETFE: SumofelectronicandthermalFreeEnergies 
Interaction and deformation energy of complex 5 are -24.23 and $1.98 \mathrm{kcal} \mathrm{mol}^{-1}$. The values show that the interaction between CNT and carbazochrome is physical adsorption. They reported that the calculated adsorption energy was approximately $-0.405 \mathrm{eV}$ with an equilibrium distance of approximately $3.167 \AA$ and stated the presence of physical absorption in the interaction [23].

Computing the free energy change of any reaction is a useful approach to assessing its thermodynamic feasibility. A large negative change in free energy as it passes from reagents to products creates a quantitative spontaneous (and exothermic) reaction. SETE in interaction for complex1 -complex6 are -2.59, 3.54, -1.72, $2.33,-7.99,-9.84,-10.13 \mathrm{kcal} \mathrm{mol}^{-1}$. It was found that the complex6 gives the strongest spontaneous (and exothermic) reaction in other configurations. It can be said that the weak intermolecular interference between CNT and Carbazochrome is an alternative, and the CNT favors the suitability of the Carbazochrome molecule as a drug, resulting in desorption [24, 25].

From the thermodynamic quantities calculated in the gas phase, it is seen that the formation of all complexes is exothermic and all except complex 2 and complex 3 are thermodynamically applicable under ambient conditions. Charge population analysis using the Mulliken approach shows that there is -0.02295 e charge flow from CNT to carbazochroma in complex1, whereas in complex2- complex6, there was a charge flow of 0.004935, 0.007103, 0.029106, 0.002562 and 0.002217 e from the carbazochrome to the CNT.

According to the Mulliken charge population analysis with the Mulliken approach, the Mulliken charge of the $\mathrm{C} 38$ atom in CNT, which was $-0.205 \overline{\mathrm{e}}$, was $-0.196 \mathrm{e}$ in complex1. The mulliken charge, which was $-0.815 \overline{\mathrm{e}}$ in carbazochrome, was $-0.796 \overline{\mathrm{e}}$ in complex1 The negative charge densities of the C38 and N1 atoms in the interaction of CNT and carbazochrome show that they decrease to $0.009 \overline{\mathrm{e}}$ and $0.019 \overline{\mathrm{e}}$, respectively. However, the fact that there is a charge density flow from CNT to carbazochroma during the interaction indicates that the charge densities are distributed over other atoms.

In complex5, which has the highest adsorption and interaction energy in the studied complexes, the charge transfer from carbazochrome to CNT was $0.002562 \overline{\mathrm{e}}$, and in this interaction, the Mulliken charge density of C38 decreased to $0.027 \mathrm{e}$ and the $\mathrm{N} 4$ and $\mathrm{O} 2$ atoms increased to 0.034 and $0.043 \mathrm{e}$. It shows that it is distributed over other atoms. In order to clarify the binding nature in these complexes, we evaluated the total electron density maps of carbazochrome, CNT and complexes. Regions with high electron density are shown in red and yellow. In the Carbazochrome molecule, it is seen that the phenyl group has a negative potential, while in the complexes it is concentrated in the binding groups, as can be seen in Fig. 2.

\section{Topology analysis:}

Topology Analysis based on Bader's QTAIM method has been employed with MULTIWFN program. It has been applied for the selected complexes with the strong interaction energy and more negative adsorption energy values given in Table 1. Thus, the property of intermolecular interactions between CNT and drug is determined in terms of electron densities at bond critical points (BCPs).

In QTAIM analysis, mostly, laplacian $\nabla^{2} \rho(r)$ characteristics and the electron density of $\rho(r)$ and are widely used to understand the bonding interactions property. However, the total energy density of $\mathrm{H}(\mathrm{r})$ and $|V(r)| / G(r)$ are more remarkable parameters on bonding characteristics. It has been noted that for weak and medium-strength hydrogen bonding and van der waals interactions due to the $\nabla^{2} \rho(r)>0, H(r)>0,|V(r)| / G(r)<1$. The strong hydrogen bonds as the intermediate type of interaction related to $\nabla^{2} \rho(r)>0, H(r)<$ 
$0,1<|V(r)| / G(r)<2$. The covalent bonding characteristics correspond to $\nabla^{2} \rho(r)<0, H(r)<$ $0,|V(r)| / G(r)>2$. The calculated parameters related to QTAIM are represented in Table 3. All the BCP parameters for the selected two complexes are given in Table 3.

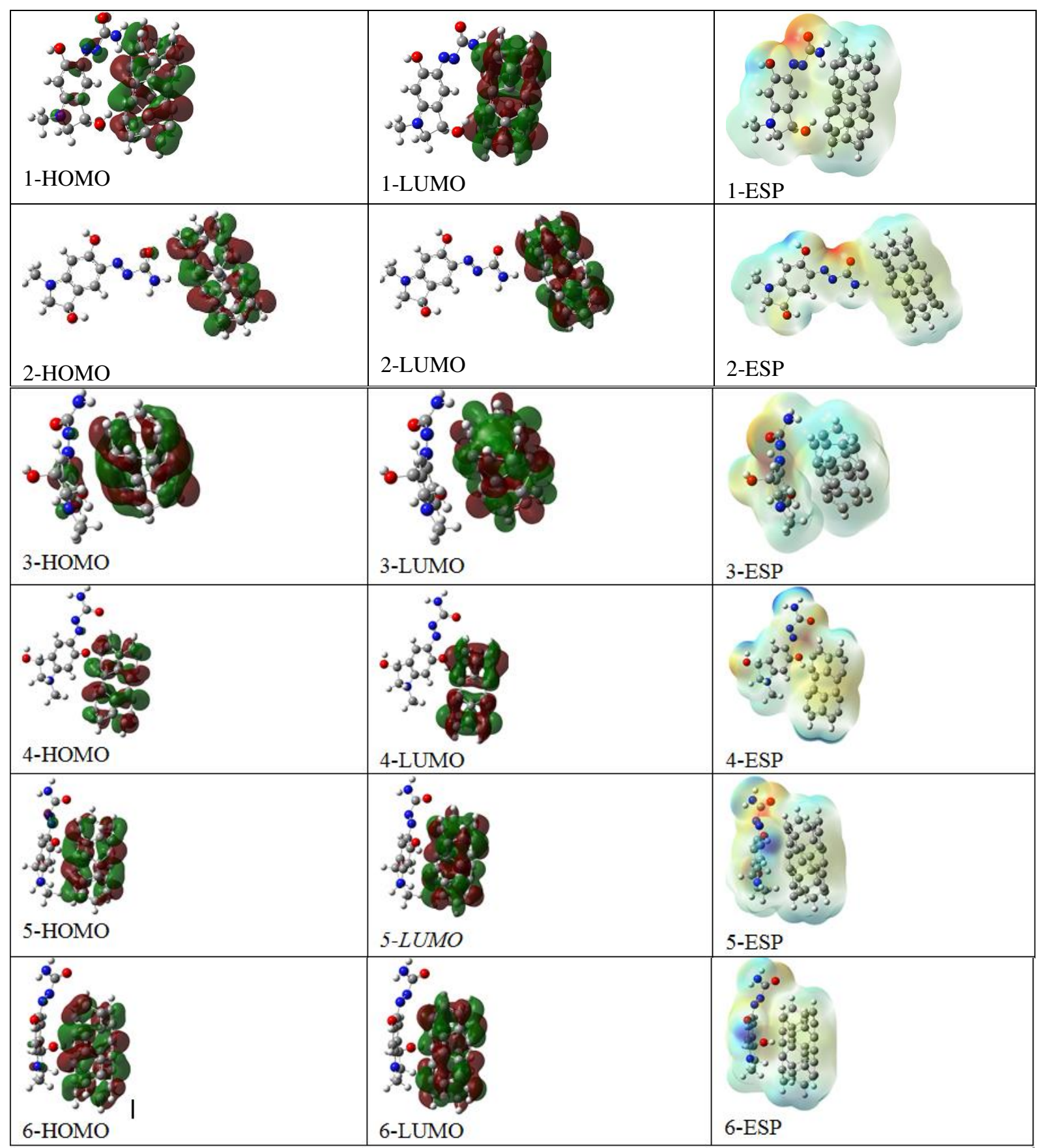

Fig. 2 HOMO, LUMO and ESP form of carbazochrome complexes (complex1-complex6)

The computed molecular topographical map of complex 1 and complex 5 with critical points and atom labels are also illustrated in Fig 3. 
Table 3 The QTAIM parameters of selected complexes at the BCPs. $(\rho(r)),\left(\nabla^{2} \rho(r)\right), \mathrm{y}(\mathrm{G}(\mathrm{r})),(\mathrm{V}(\mathrm{r}))(\mathrm{H}(\mathrm{r}))$, the ratio $\left|V_{B C P}\right| / G_{B C P}$

\begin{tabular}{clcccccc}
\hline \multirow{5}{*}{$\begin{array}{c}\text { BCP } \\
\text { Drug- } \\
\text { CNT }\end{array}$} & $\rho_{B C P}$ & $\nabla^{2} \rho_{B C P}$ & $\mathrm{G}_{\mathrm{BCP}}$ & $\mathrm{V}_{\mathrm{BCP}}$ & $\mathrm{H}_{\mathrm{BCP}}$ & $\frac{\left|V_{B C P}\right|}{G_{B C P}}$ \\
\hline Complex & O58-C3 & 0.0089953 & 0.02864881 & 0.00637412 & -0.00558601 & $0.78810 \mathrm{E}-3$ & 0.876359 \\
$\mathbf{1}$ & H60-C10 & 0.0064457 & 0.20480840 & 0.00408164 & -0.00304308 & 0.00103856 & 0.745553 \\
& H51-C9 & 0.0086487 & 0.02789399 & 0.00568965 & -0.00440579 & 0.00128385 & 0.774353 \\
& H62-C18 & 0.0168489 & 0.05058477 & 0.01218945 & -0.01173273 & $0.45673 \mathrm{E}-03$ & 0.962531 \\
& H43-C32 & 0.0058093 & 0.01999599 & 0.00391383 & -0.00282866 & 0.00108517 & 0.722735 \\
& H69.C23 & 0.0084599 & 0.02777012 & 0.00574151 & -0.00454049 & 0.00120102 & 0.790818 \\
& N45-C32 & 0.0095906 & 0.02925069 & 0.00637501 & -0.00543734 & $0.93766 \mathrm{E}-03$ & 0.852916 \\
Complex & N41-C38 & 0.0092201 & 0.02648873 & 0.00598239 & -0.00534261 & $0.63979 \mathrm{E}-03$ & 0.893055 \\
$\mathbf{5}$ & O66-C35 & 0.0062120 & 0.02545403 & 0.00519646 & -0.00402941 & 0.00116705 & 0.775415 \\
& O66-H37 & 0.0127652 & 0.04707427 & 0.01073079 & -0.00969302 & 0.00103777 & 0.903290 \\
& N62-C38 & 0.0151947 & 0.05264539 & 0.01149667 & -0.00983199 & 0.00166467 & 0.855204 \\
& O61-C31 & 0.0075404 & 0.02455781 & 0.00533510 & -0.00453076 & $0.80435 \mathrm{E}-03$ & 0.849235 \\
& O61-C23 & 0.0065634 & 0.01884586 & 0.00399188 & -0.00327229 & $0.71958 \mathrm{E}-03$ & 0.819738 \\
& C49-C23 & 0.0062352 & 0.01745302 & 0.00369054 & -0.00301782 & $0.67272 \mathrm{E}-03$ & 0.817719 \\
& C48-C23 & 0.0066979 & 0.01813392 & 0.00384244 & -0.00315139 & $0.69104 \mathrm{E}-03$ & 0.820155 \\
& H58-C24 & 0.0121040 & 0.03648692 & 0.00816775 & -0.00721377 & $0.95398 \mathrm{E}-03$ & 0.883201 \\
& H47-C24 & 0.0059264 & 0.01831454 & 0.00372119 & -0.00286374 & $0.85745 \mathrm{E}-03$ & 0.769577 \\
\hline
\end{tabular}
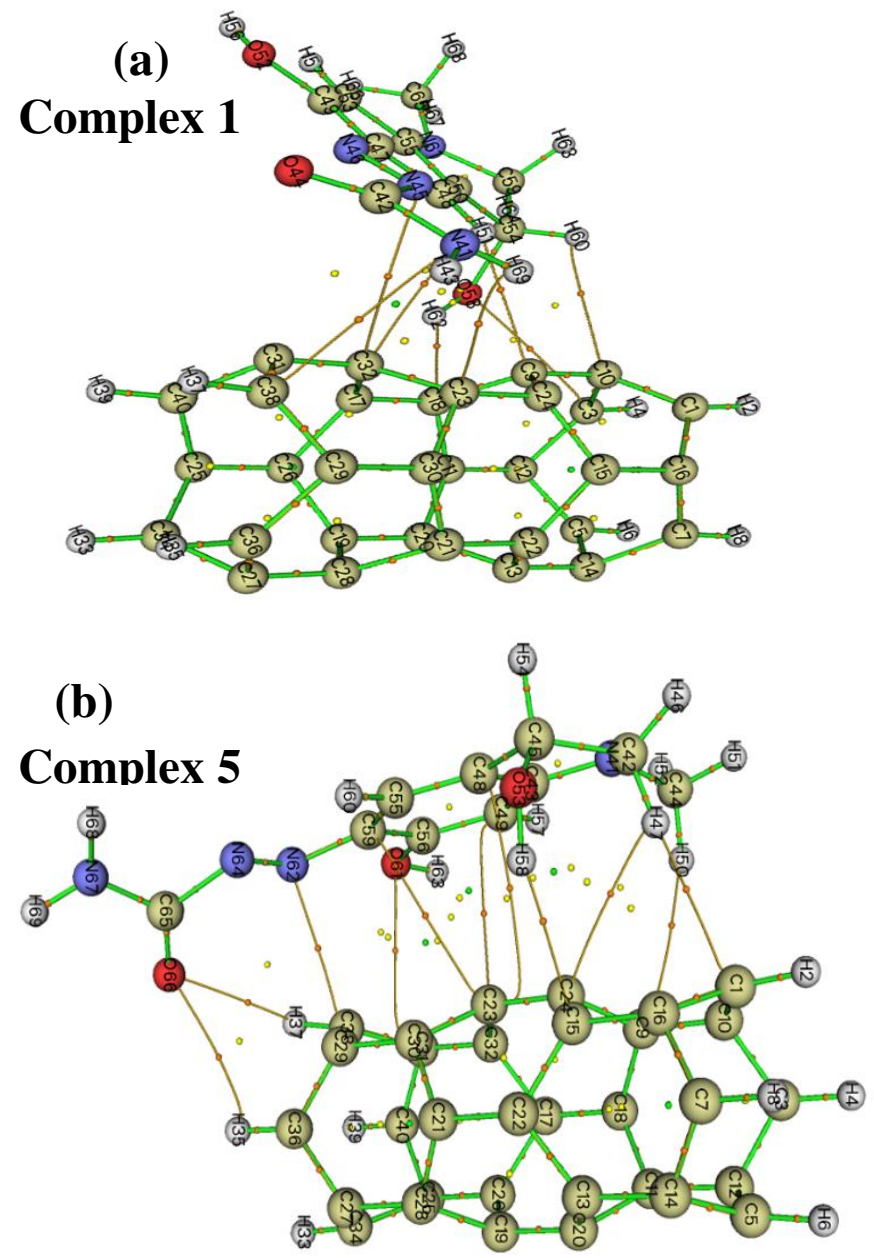

Fig. 3 The computed molecular topographical map of a) complex 1 and b) complex5 with all critical points 
According to Table 3, the highest electron density at BCP between the nitrogen atom (N62) of the drug and the carbon atom (C38) of the CNT, which indicates more accumulation of charge from the computational results presented in Table2, the all interactions in the both complex structures indicate the typical electrostatic interactions according to QTAIM callsification with the toplogy parameters as $\nabla^{2} \rho(r)>0, H(r)>$ $0,|V(r)| / G(r)<1$.

QTAIM analysis shows that the majority of the interactions of drug with the surface of CNT can be defined as non-covalent nature. One of the important measurements of chemical stability is the energy gap between HOMO and LUMO, and if a molecule is little or no HOMO - LUMO gap, it can be chemically reactive.

Atomic and bond critical points are presented by atom labels and orange spheres, respectively. The cage and ring critical points due to green and yellow circles. The lines are bond paths

Table 4 and Table 5 summarize HOMO-LUMO energies and the parameters like such as hardness ( $\eta$ ), softness $(\sigma)$, electronegativity $(\chi)$, the maximum amount of electronic charge transfer $\Delta \mathrm{N}_{\text {maks, }}$, Chemical potential $(\mu)$ and electrophilicity index $(\omega)$, nucleofugality, electrofugality related to $\mathrm{E}_{\text {HOMO }}$ and $\mathrm{E}_{\mathrm{LuMO}}$, and, thermal energy, heat capacity and entropy values of Carbazochrome, CNT and complex1-complex6 calculated with M062X/6-31g(d) level.

Table $4 \mathrm{E}_{\mathrm{HOmO}}, \mathrm{E}_{\mathrm{LumO}}, \Delta \mathrm{E}_{\mathrm{g}}$, hardness, softness, the maximum amount of electronic charge $\operatorname{transfer}_{\mathrm{s}}$ ) of Carbazochrome, CNT and complex1-complex6 calculated with M062X functional and 6-31g (d) basis set. \% $\Delta$ $\mathrm{E}_{\mathrm{g}}$ represents the percentage of band gap of complex with respect to the bare CNT

\begin{tabular}{|c|c|c|c|c|c|c|c|}
\hline Molecules & $\begin{array}{l}\mathrm{E}_{\text {HOMO }} \\
(\mathrm{eV})\end{array}$ & $\begin{array}{l}\text { E }_{\text {LUMO }} \\
(\mathrm{eV})\end{array}$ & $\begin{array}{l}\Delta \mathrm{E}_{\mathrm{g}} \\
(\mathrm{eV})\end{array}$ & $\% \Delta \mathrm{E}_{\mathrm{g}}$ & $\begin{array}{l}\eta \\
(\mathrm{eV})\end{array}$ & $\begin{array}{l}\sigma \\
\left(\mathrm{eV}^{-1}\right)\end{array}$ & $\Delta \mathrm{N}_{\text {maks }}$ \\
\hline Carbazome & -6.753 & -0.896 & 5.856 & & 2.928 & 0.171 & 1.306 \\
\hline CNT & -6.220 & -2.304 & 3.916 & & 1.958 & 0.255 & 2.177 \\
\hline 1 & -6.283 & -2.382 & 3.902 & -0.36 & 1.951 & 0.256 & 2.221 \\
\hline 2 & -6.064 & -2.158 & 3.905 & -0.28 & 1.953 & 0.256 & 2.105 \\
\hline 3 & -6.227 & -2.352 & 3.875 & -1.05 & 1.938 & 0.258 & 2.214 \\
\hline 4 & -6.077 & -2.160 & 3.917 & 0.025 & 1.958 & 0.255 & 2.103 \\
\hline 5 & -6.139 & -2.232 & 3.907 & -0.22 & 1.953 & 0.256 & 2.143 \\
\hline 6 & -6.262 & -2.346 & 3.915 & -0.02 & 1.958 & 0.255 & 2.199 \\
\hline
\end{tabular}

Table 5 Chemical potential, electronegativity, electrophilicity,, nucleofugality, electrofugality values of Carbazochrome, CNT and complex1-complex6 calculated with M062X/6-31g (d) level

\begin{tabular}{cllllll}
\hline Molecules & $\chi$ & $\mu$ & $\begin{array}{l}\omega \\
(\mathrm{eV})\end{array}$ & $\begin{array}{l}\Delta \mathrm{E}_{\mathrm{n}} \\
(\mathrm{eV})\end{array}$ & $\begin{array}{l}\Delta \mathrm{E}_{\mathrm{e}} \\
(\mathrm{eV})\end{array}$ & $\mathrm{K}$ \\
\hline Carbazome & 3.825 & -3.825 & 2.498 & 0.137 & 7.786 & \\
$\mathrm{CNT}$ & 4.262 & -4.262 & 4.639 & 1.356 & 9.880 & \\
1 & 4.333 & -4.333 & 4.811 & 1.454 & 10.119 & $1.82 \times 10^{1}$ \\
2 & 4.111 & -4.111 & 4.328 & 1.193 & 9.415 & $2.53 \times 10^{-3}$ \\
3 & 4.290 & -4.290 & 4.749 & 1.428 & 10.007 & $1.60 \times 10^{-3}$ \\
4 & 4.118 & -4.118 & 4.330 & 1.191 & 9.428 & $1.37 \times 10^{4}$ \\
5 & 4.186 & -4.186 & 4.484 & 1.275 & 9.646 & $1.1310^{6}$ \\
6 & 4.304 & -4.304 & 4.731 & 1.406 & 10.014 & $8.95 \times 10^{5}$ \\
\hline
\end{tabular}


The isolated carbazochrome has a large HOMO-LUMO energy gap of $5.856 \mathrm{eV}$, where $\mathrm{E}_{\mathrm{HOMO}}$, is $-6.753 \mathrm{eV}$, and the $\mathrm{E}_{\mathrm{LUMO}}$, is $-0.896 \mathrm{eV}$. The isolated CNT has $3.916 \mathrm{eV}$ HOMO-LUMO energy gap, where the HOMO energy is $-6.220 \mathrm{eV}$, and the LUMO energy is $--2.304 \mathrm{eV}$. As can be seen in Table 3, after adsorption of Carbazochrome on CNT, the HOMO-LUMO energy gap of system decrease to 3.902, 3.905, 3.875, 3.907, $3.915 \mathrm{eV}$, respectively for complex1- complex6. however, increase slightly to 3.917 for complex4.

In order to understand the sensing mechanism of the CNT to drug, the variation of $\Delta \mathrm{E}_{\mathrm{g}}$ gap during the adsorption process is taken into account by the following equation.

$$
\% \Delta E_{g}=100 x\left(\Delta E_{g 2}-\Delta E_{g 1}\right) / \Delta E_{g 1}
$$

Where $\Delta E_{g 1}$ and $\Delta E_{g 2}$ are the $\Delta E_{g}$ values of CNT and complex, respectively.

Sensors are concerned with the change of their electrical conductivity after drug adsorption due to electron exchange between the drug and the sensor. In this line, the drug sensitivity of CNT is based on the HOMO and LUMO energies.

The percentage change values, of $\% \Delta E_{g}$ for each complex are given in Table3. As reported energies of $\mathrm{E}_{\mathrm{HOMO}}$ and $\mathrm{E}_{\mathrm{LUMO}}$ and energy gap (Eg) in Table 3, the electronic properties of CNT are affected by the adsorption of Carbazochrome drug. The decreasing order of percentage change values are obtained for complex3, complex1, complex2, complex 5 and complex 6 as $1.05 \%, 0.36 \%, 0.28 \%, 0.22 \%, 0.022 \%$ but for complex 4 is about $\%$ 0.025 by increasing.

The lower Eg values indicate higher electrical conductivity, reactivity and sensitivity. Therefore, decreased the $\mathrm{E}_{\mathrm{g}}$ through adsorption of Carbazochrome drug indicate the CNT can detect the drug. Except complex4, all studied geometries of complex structures can be detected the drug material. The density of states (DOS) diagram of each complex was also calculated in order to better understanding the stability of the system. The change in $\mathrm{E}_{\mathrm{g}}$ by DOS analysis can be confirmed as shown in Fig. 4. 

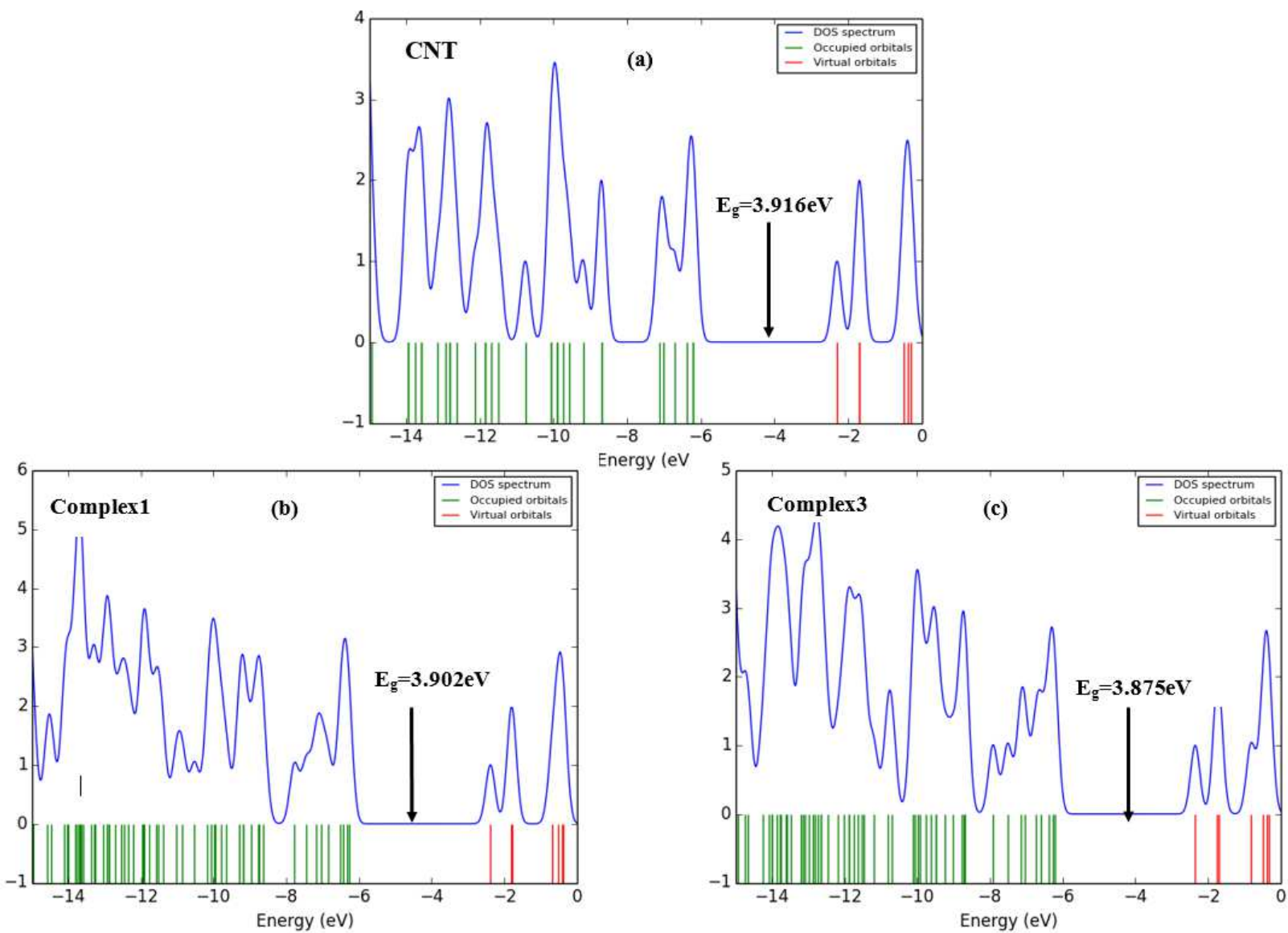

Figure 4. TDOS graphs for a) pristine CNT, b) complex1 and c) complex3 structures

The DOS diagrams of CNT and selected complexes are calculated in the energy range of -15 to $0 \mathrm{eV}$ have been shown in Figure 4. In these plots, we have selected the complexes with the significant $\% \Delta E_{g}$ values given in Table3, as complex3 and complex1.

The ionization potential of the system may decrease when carbazochrome is adsorbed (complex2, complex4, and complex5) or increase (complex1, complex3 and complex6) depending on the adsorption site; but these variations are not noticeable

The stability of chemical compounds against deformation in an electric field can be related by its hardness. The hardness of CNT being $1.958 \mathrm{eV}$ is decreased to $1.951,1.953,1.951,1.938,1.953 \mathrm{eV}$ for complex1complex3 and complex 5 and it is the same for complex 2 and complex 5 . When the hardness values are compared, the stability of complex4 and complex6 is the same as that of the CNT.

ELF current-densities image obtained from ab initio studies of Carbazochrome carbon nanotube as drug delivery nanocarrier for anti-bleeding drug are represented in (Fig. 5) and the currents consist of HOMO contributions (Table. 4). 


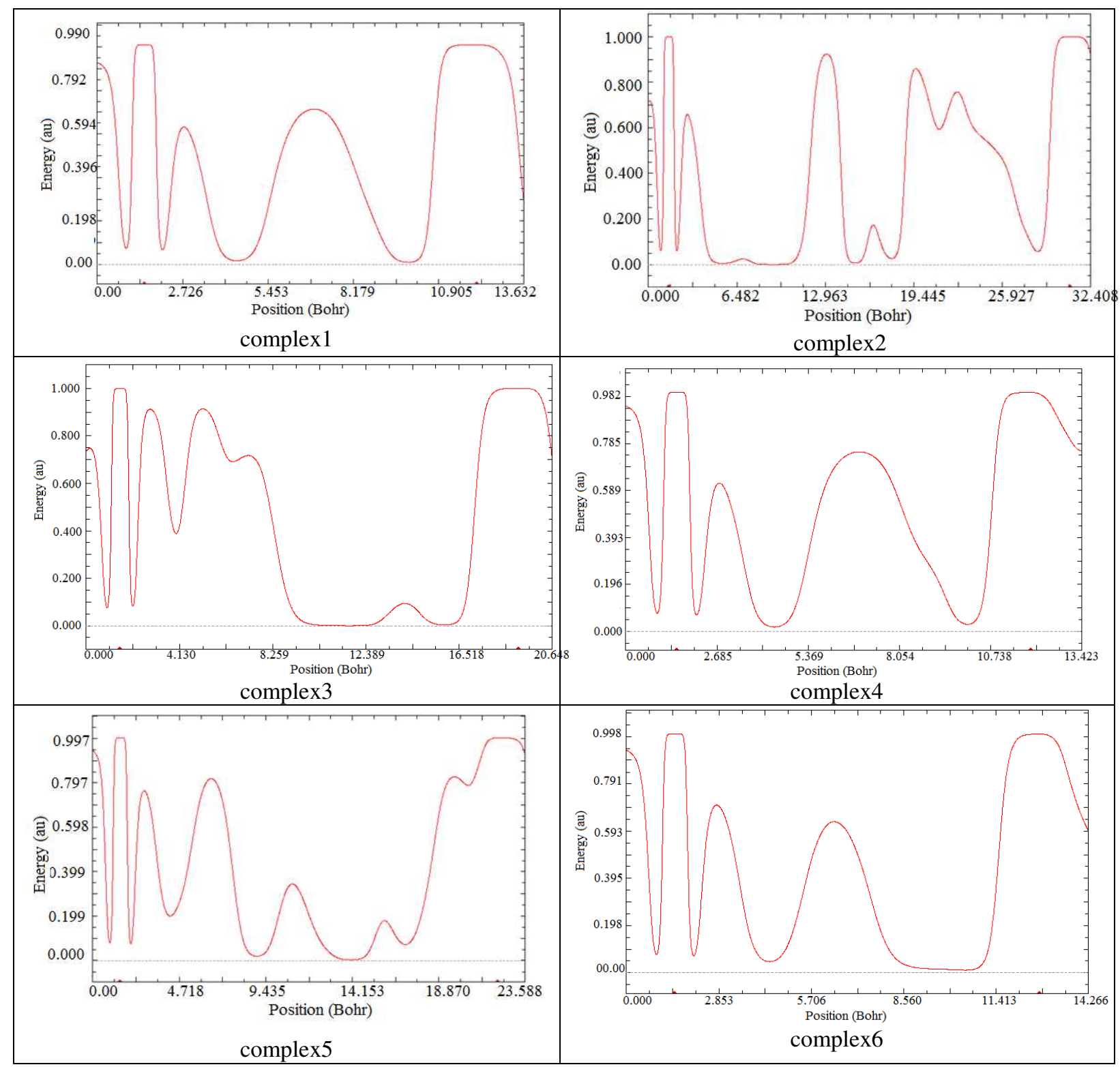

Fig. 5 ELF current-density map from ab initio calculations of Carbazochrome carbon nanotube as drug delivery nanocarrier

In a ring having " $4 \mathrm{n}$ " electrons, HOMO-LUMO is derived from a split degenerate $(\lambda=\mathrm{n})$, whereas in a ring with a " $4 n+2$ " system such as Carbazochrome, HOMO-LUMO is associated with " $n+1$ " and " $n$ ". $\lambda$ is zero when $n=4$. The dia-tropic contribution and the para-tropic contribution consist of transitions with $\Delta \lambda=+1$ and $\Delta \lambda=0$, respectively [26, 27]. In Huckel's theory, $\{\pi\}$ energies are calculated using coulomb and resonance parameter $\{\beta\}[28]$. Therefore, for higher symmetry $4 n+2$ systems, HOMO-LUMO transitions cause to diatropic currents. For the " 4 n" ring (subsymmetry), the HOMO-LUMO transition leads to para-tropical contribution and the second HOMO-LUMO+1 transition cause to tropic contributions [28].

To understand in depth the role of interacting atoms, the chemical bonds of the complexes, TDOS, PDOS and OPDOS were performed with the Multiwfn package. In the TDOS map, each individual vertical line matches to MO, and the dashed line highlights the place of the HOMO. In TDOS, the curve is simulated according to the distribution of MO energy levels. As can be seen from the figure, the region around -0.40 a.u. on the negative side clearly has a greater state density than the other regions. Fragment 1 was defined as the atoms of nanotube 
and fragment 2 was defined of carbazochrome molecule and fragment 3 was defined as atoms that interact in complex formation to check the effect of interaction atoms to bond formation corresponds to TDOS and PDOS, and the right axis corresponds to OPDOS which gives information related to bonding, anti-bonding and nonbonding interactions of two orbitals, atoms or groups. The vertical dashed line defines the position of the HOMO orbital. The dashed line for OPDOS lines i.e. values greater or less than zero of the corresponding state density indicate MOs with or without a contribution to the corresponding bonding, respectively. As can be seen from the Fig 6, the TDOS in the most stable complex is precisely the superposition of the non bonded carbazochrome molecule and the PDOS of the nanotube.

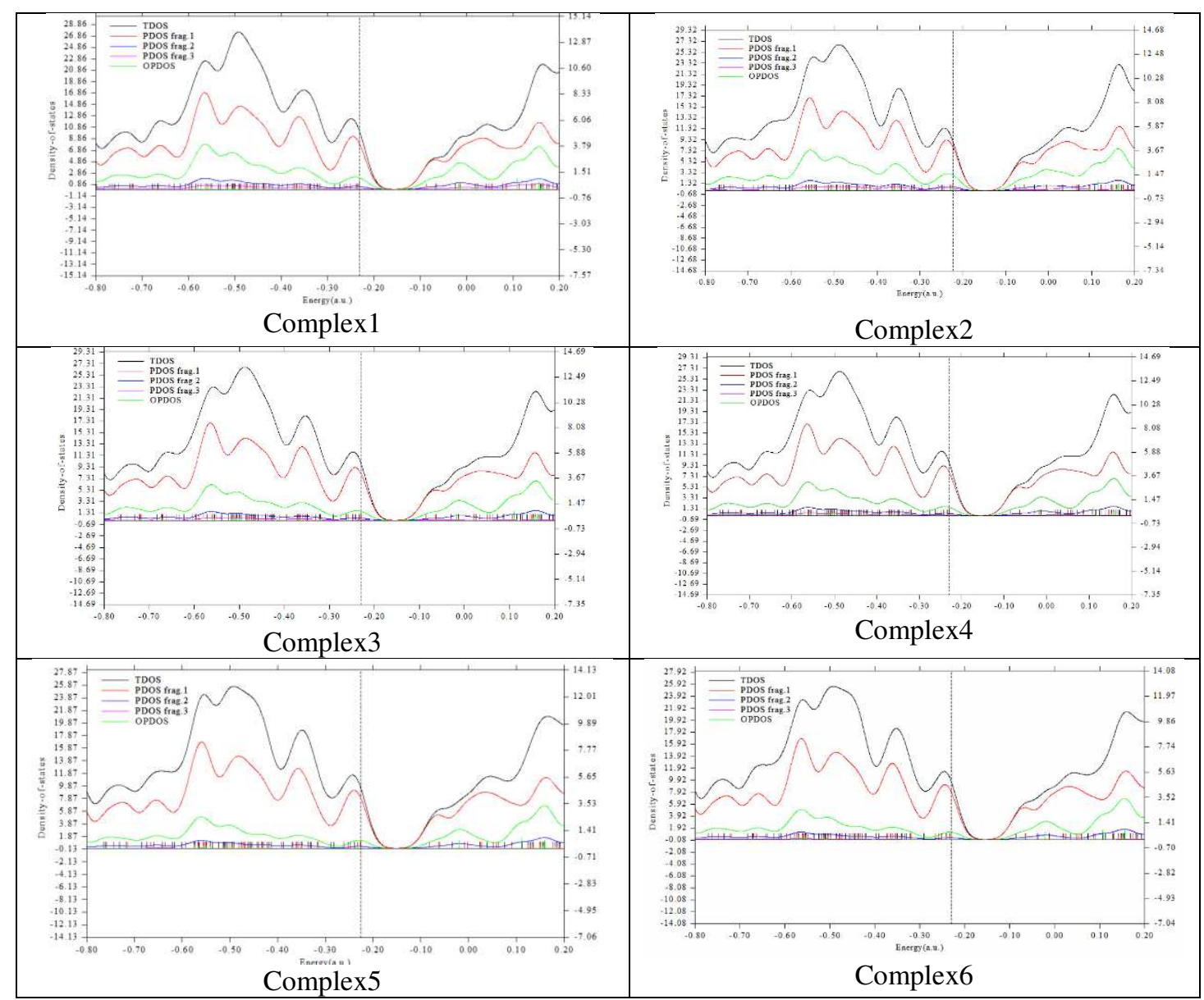

Fig. 6. The TDOS, PDOS, and OPDOS curves for complex1-complex6

The graph of TDOS/PDOS/OPDOS for complexes are given in Fig. 6. The left axis compared with the carbazochrome molecule adsorbed in the nanotube complex, the DOS plot of a free carbazochrome molecule defines that after the adsorption of the carbazochrome molecule, the DOS of the nanotube increases more, causing to a notable increase in the characteristic properties of the DOS plot. These result show that the electronic properties of the complexes varies with the interaction of carbazochrome and nanotube.

When the green OPDOS curve corresponding to the bond formed by the carbazochrome molecule and pristine CNT interaction is examined, it shows that the carbon orbitals formed from this interaction are very important for the stabilization of the complexes, since OPDOS has a large positive value in these intervals. When the green OPDOS curve corresponding to the bond formed by the carbazochrome molecule and nano-tube interaction is 
examined, it shows that the orbitals formed from this interaction are very important for the stabilization of the complexes, since OPDOS has a large positive value in these intervals.

The chemical hardness $(\eta)$ and chemical potential $(\mu)$ for the investigated structures are the parameters used to predict the reactivity of the molecules. Since compounds with lower chemical hardness and higher chemical potential values will be more reactive, the electron transfer required for the implementation of the chemical reaction can be easier [29]. As can be seen, in complex 2 and complex5, when carbazochrome is adsorbed to CNT surfaces, the hardness decreases and the chemical potential increases in both configurations, indicating that the reactivity of carbazochrome increases after its adsorption to the CNT surface [30].

The global electrophilicity index measures the total ability to attract electrons. CNT can be accepted as a strong electrophile due to possessing a high electrophilicity index at B3LYP (4.639) and carbazochrome posses a lower electrophilicity index at B3LYP (2.498). Therefore, in the interaction of the carbazochrome with the CNT, the electron density flows from the carbazochrome to the CNT [31].

The fractional number $(\Delta \mathrm{N})$ of the electron transferred between two molecules is determined by Equation 12 . Electron transfer parameter determines the direction of spontaneous electron flow, as well as the fraction of electrons transferred from system A to system B [25, 32]

$$
\Delta N=\frac{\mu_{B}-\mu_{A}}{\eta_{A}+\eta_{B}}
$$

Where, $\eta_{\mathrm{A}}$, and $\eta_{\mathrm{B}}$ chemical hardness of the acceptor $(\mathrm{A})$ and donor (B) systems, and $\mu_{\mathrm{A}}$ is chemical potential of the acceptor $(\mathrm{A})$ and $\mu_{\mathrm{B}}$ is those of donor (B) systems. If $\Delta \mathrm{N}$ is positive, the charge transfer occurs spontaneously from $\mathrm{B}$ to $\mathrm{A}$, and if negative, the charge transfer occurs in the opposite direction. $\Delta N$ value of 0.09 in the equation $\Delta N=\frac{\mu_{\text {carbazole }}-\mu_{C N T}}{\eta_{\text {carbazole }}+\eta_{C N T}}$ supports that the charge flow from Carbazome to CNT.

The thermodynamic equilibrium constants of the reaction are also calculated by placing the values of Gibbs free energy changes in the following equation. In this formula, $\mathrm{R}$ denotes the ideal gas constant and $\mathrm{T}$ the temperature. As can be seen from the Table 4, Complex 5 has the largest $\mathrm{K}$ value among the studied complexes.

$$
K=\exp \left(-\frac{\Delta G_{a d s}}{R T}\right)
$$

The results of the dipole moments $(\mu)$, static and dynamic polarizability $(\alpha)$, anisotropic polarisability, and first hyperpolarizability $(\beta)$ of carbazochrome, CNT and complexes obtained by the interaction of different functional groups with M062X6-31G (d) are given in Table 6.

The total electric dipole moments composed of electric dipole moment components $\left(\mu_{\mathrm{x}}, \mu_{\mathrm{y}}, \mu_{\mathrm{z}}\right)$, the averaged (isotropic) dipole polarizabilities $(\alpha)$, anisotropy polarizability $(\Delta \alpha)$, and total first static hyperpolarizability from the polarizability components are evaluated as follows respectively [33, 34].

$$
\begin{aligned}
& \mu=\left(\mu_{x}^{2}+\mu_{y}^{2}+\mu_{z}^{2}\right)^{1 / 2} \\
& \alpha=\frac{1}{3}\left(\alpha_{x x}+\alpha_{y y}+\alpha_{z z}\right) \\
& \Delta \alpha=\left[\frac{\left(\alpha_{x x}-\alpha_{y y}\right)^{2}+\left(\alpha_{y y}-\alpha_{z z}\right)^{2}+\left(\alpha_{z z}-\alpha_{x x}\right)^{2}+6\left(\alpha_{x z}^{2}+\alpha_{x y}^{2}+\alpha_{y z}^{2}\right)}{2}\right]^{1 / 2} \\
& \beta_{x}=\beta_{x x x}+\beta_{x y y}+\beta_{x z z} \\
& \beta_{y}=\beta_{y y y}+\beta_{y z z}+\beta_{y x x} \\
& \beta_{z}=\beta_{z z z}+\beta_{z x x}+\beta_{z y y} \\
& \beta=\left(\beta_{x}^{2}+\beta_{y}^{2}+\beta_{z}^{2}\right)^{1 / 2}
\end{aligned}
$$


Table 6 The dipole moment, polarizability, anisotropic polarizability and hyperpolarizability values of carbazochrome, pristine CNT, and complex1-complex6 molecules calculated with the M062X/6-31g(d) level

\begin{tabular}{cllrrl}
\hline Complex & \multicolumn{1}{c}{$\mu$} & $\begin{array}{c}\alpha \\
\mathrm{D}\end{array}$ & $10^{-24} \mathrm{~cm}^{3}$ & Kappa & \multicolumn{2}{c}{$\begin{array}{c}\beta \\
10^{-30} \mathrm{~cm}^{5} \mathrm{esu}^{-1}\end{array}$} \\
\hline 1 & 8.57 & 70.63 & 0.02986 & 41.17 & 16.42 \\
2 & 14.02 & 74.93 & 0.04623 & 54.48 & 62.49 \\
3 & 13.67 & 70.36 & 0.01064 & 40.14 & 22.96 \\
4 & 4.81 & 71.25 & 0.03356 & 53.97 & 15.56 \\
5 & 5.04 & 69.25 & 0.02567 & 44.32 & 13.03 \\
6 & 21.63 & 69.07 & 0.02570 & 45.42 & 15.10 \\
Carbazochrome & 11.89 & 23.54 & 0.11460 & 24.19 & 25.42 \\
CNT & 0.00 & 47.71 & 0.08584 & 41.93 & 0.01 \\
\hline
\end{tabular}

a.u. $($ Bohr-electron $)=2.541765$ Debye, Polarizability: $(\alpha) 1$ a.u. $=0.148184709 .10^{-24} \mathrm{~cm}^{3}$;

First-hyperpolarizability $(\beta): 1$ a.u. $=8.639418 .10^{-33} \mathrm{~cm}^{5} \mathrm{esu}^{-1}$

A dipole moment is a mathematical calculation dealing with unequal distribution of charge in a compound. In other words, the higher the dipole moment of a compound, the more polar the compound is. On the other hand, polarizability is the tendency of a compound to form a dipole when an external electric field is encountered.

In an applied electric field, the energy of a system is a function of the electric field. The first hyperpolarizability is the third order tensor with a $3 \times 3 \times 3$ matrix. The 27 components of the 3-dimensional matrix can be decreased to 10 components by means of Kleinman symmetry [35]. The $\mu$ of pure model CNT is 0 and the symmetry is very high, therefore its corresponding $\beta$ value is very small $\left(0.01 \times 10^{-30} \mathrm{~cm}^{5} \mathrm{esu}^{-1}\right)$. The value of the dipole moment increases in the adsorbed configurations relative to the free CNT (0.00 Debye), which can be attributed to the the perturbation in the electron density. The dipole moment of complex1 (8.57), complex4 (4.81) and complex5 (5.04) is smaller than that of the single drug and the dipole moment of Complex2 (14.02), complex3 (13.67) and complex6 (21.63) is higher than that of the single carbazochrome which shows that adsorption of Carbazochromer on the CNT in these three complexes enhances the polarity of the system that is a good feature for drug delivery in biological environments [36].

When carbazochrome is adsorbed to CNT, the symmetry is broken. The $\mu$ and $\beta$ values both increased, and the variation of $\beta$ value is very apparent. The $\beta$ value of complex1-complex 6 are $16.42,62.49,22.96,15.56,13.03$, $15.10 \times 10^{-30} \mathrm{~cm}^{5} \mathrm{esu}^{-1}$. When we compare the different interaction ways, the hyperpolarisability value of complex1, which is formed by the interaction of CNT and carbazochrome with the carbonyl group, is the largest of the complexes formed by the interaction of CNT and carbazochrome with the other groups It has been reported that a large initial hyperpolarizability $\left(4.187944 \times 10^{-30} \mathrm{esu}\right)$ value is a prerequisite for it to behave like a good NLO material [37].

\section{Recovery Time}

We have calculated recovery time of the drug from the studied CNT surfaces. It is known that the recovery time gives an information about the time interval (short or long) of the deformation process. It can be predicted as follows

$$
\tau=v^{-1} \exp \left(-\frac{E_{a d s}(B S S E)}{k T}\right)
$$

Where, $v$ is the attempt frequency, $\mathrm{E}_{\mathrm{ads}}$ is the BSSE corrected adsorption energy, $\mathrm{k}$ is the Boltzmann constant and $\mathrm{T}$ is temperature. If the vacuum UV light $\left(v \sim 3 \times 10^{16} s^{-1}\right)$ is applied to recover of the drug from the studied surfaces. The CP corrected binding energy values are used in the above Eq. (14) that obtained for complex1- 
complex6. $-13.59 \mathrm{kcal} \mathrm{mol}^{-1},-7.43 \mathrm{kcal} \mathrm{mol}^{-1},-6.55 \mathrm{kcal} \mathrm{mol}^{-1},-15.177 \mathrm{kcal} \mathrm{mol}^{-1},-21,036 \mathrm{kcal} \mathrm{mol}^{-1},-21,069$

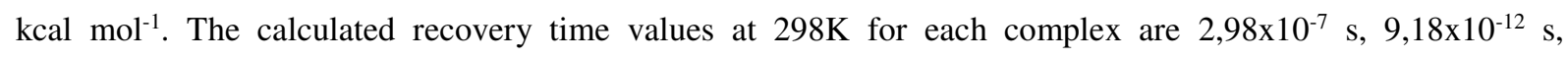
$2,098 \times 10^{-12} \mathrm{~s}, 4,348 \times 10^{-6} \mathrm{~s}, 0,0849 \mathrm{~s}, 0,0897 \mathrm{~s}$ for complex 1 -complex6, respectively. Those recovery time values for complex1-complex3 are too short, other values for complex5 and complex6 indicate that desorption of drug from the CNT surface is possible. Thus the CNT surface can be a proper sensor material for carbazole drug under UV light frequency and room temperature.

In addition under the UV-Light frequency $\left(v \sim 3 \times 10^{12} s^{-1}\right)$, which is used for the drug $12 s^{-1}$ desorption from nanosurfaces [38]. Here, the recovery time of $849 \mathrm{~s}$ for complex 5 was calculated under the UV-Light. It is an acceptable value for the desorption process of carbazochrome drug from CNT surfaces.

\section{Conclusion}

In this study, we perused the Carbazochrome molecule adsorption on the CNT by various functional groups performing DFT calculations provided valuable information on structural properties, adsorption energy, orientation and lcharge transfer between CNT and Carbazochrome drug in CNT / Carbazochrome complexes. QTAIM analysis was implemented to consider the characteristics of the intermolecular interactions.

The formation of all gas-phase complexes is exothermic, and all are thermodynamically feasible under ambient conditions except for complex 2 and complex3. The results indicated that when carbazochrome adsorbed on CNT the hyperpolarizability for adsorbed nanotubes increase many times, which indicate that organic chromophore adsorbed to nanotube enhance the nonlinear optical properties. It has been shown that the interaction with different functional groups changes the geometric structure, charge distribution and affects the electrical properties, dipole moment and polarization. On this line, based on the percentage values of band gap of complexes with respect to the bare CNT, the CNT $(4,0)$ can be used to detect Carbazochrome drug as an electrochemical sensor. Furthermore, first-order hyperpolarizability of the complex 2 shows that is an attractive object for future studies of nonlinear optical properties. The nature of the formed bonding mode analysis along the reaction pathways was further approved via electron localization function (ELF) calculations. The total, partial, and overlap population density states were calculated and found that the orbitals formed from this interaction are very important for the stabilization of the complexes, since OPDOS has a large positive value in these intervals.

Authors' contributions The first draft of the manuscript was written by Fatma Kandemirli and Hakan S. Sayiner and all authors commented on previous versions of the manuscript. All authors contributed to the study conception and design. Material preparation, data collection. Research design, the acquisition, analysis and interpretation of data. All authors read and approved the final manuscript.

Funding The authors acknowledge financial support from Kastamonu University

Availability of data and material All data generated or analyzed during this study are included in this published article.

Code availability N/A.

\section{Declarations}

Conflict of interest The authors declare no competing interests

\section{References}

1. Verstraete, M (1977) Haemostatıc Drugs A critical appraisal 
2. Dykes ER, Anderson R (1961) Carbazochrome Salicylate as a Systemic Hemostatic Agent in Plastic Operations A Clinical Evaluation. JAMA 177(10):716-717. doi: 10.1001/jama.1961.73040360021012e. PMID: 13725379.

3. Meenashi R, Jayalakshmi P, Jothi B, Selvaraju K, Stephen AD (2019) Ab Initio Prediction of Stable Crystal Structure of Procarbazine Molecule. IJRAR 6(2):25-34. doi:10.26713/JAMCNP.V6I1.1114

4. Olanow CW, Stern MB, Sethi K (2009) The scientific and clinical basis for the treatment of Parkinson disease. Neurology 26:72(21 Suppl 4):S1-136. doi:10.1212/WNL.0b013e3181a1d44c

5. Vashist SK, Zheng D, Pastorin G, Al-Rubeaan K, Luong JH, Sheu FS (2011) Delivery of drugs and $\begin{array}{llllll}\text { biomolecules } \quad \text { using } & \text { carbon } & \text { (13):4077-4097. }\end{array}$ https://doi.org/10.1016/j.carbon.2011.05.049

6. Wright LR, Rothbard JB, Wender PA (2003) Guanidinium rich peptide transporters and drug delivery. Curr. Proteomics 4:105-124. doi: 10.2174/1389203033487252.

7. Langel U (2006) Cell-penetrating peptides: processes and applications, , CRC Press, Boca Raton, Fla, USA, 2nd edition

8. Berber S, Kwon YK, Tománek D (2000) Unusually high thermal conductivity of carbon nanotubes. Phys. Rev. Lett 84:4613-4616. https://link.aps.org/doi/10.1103/PhysRevLett.84.461

9. Shi Kam NW, Jessop TC, Wender PA, Dai H (2004) Nanotube molecular transporters: internalization of carbon nanotube-protein conjugates into Mammalian cells J. Am. Chem. Soc 9:126(22), 6850-6851. doi: $10.1021 / \mathrm{ja} 0486059$

10. Zare K, Shadmani N (2013) Comparison of drug delivery systems: Nanotube and pSulphonatocalix[4]arene, by Density Functional Theory. J Nanostructure Chem 3:72 1-6. http://www.jnanochem.com/content/3/1/72

11. Yoosefian M, Jahani MA (2019) Molecular study on drug delivery system based on carbon nanotube for the novel norepinephrine prodrug, Droxidopa. J. Mol. Liq 284:258- 264 . doi: 10.1016/j.molliq.2019.04.016. doi: 10.1016/j.molliq.2019.04.016

12. Frisch MJ, Trucks GW, Schlegel HB, Scuseria GE, Robb MA, Cheeseman JR, Scalmani G, Barone V, Mennucci B, Petersson GA, Nakatsuji H, Caricato M, Li X, Hratchian HP, Izmaylov AF, Bloino J, Zheng G, Sonnenberg JL, Hada M, Ehara M, Toyota K, Fukuda R, Hasegawa J, Ishida M, Nakajima T, Honda Y, Kitao O, Nakai H, Vreven T, Montgomery JA, Jr Peralta E, Ogliaro F, Bearpark M, Heyd JJ, Brothers E, Kudin KN, Staroverov VN, Kobayashi R, Normand J, Raghavachari K, Rendell A, Burant JC, Iyengar SS, Tomasi J, Cossi M, Rega N, Millam JM, Klene M, Knox JE, Cross B, Bakken V, Adamo C, Jaramillo J, Gomperts R, Stratmann RE, Yazyev O, Austin AJ, Cammi R, Pomelli C, Ochterski JW, Martin RL, Morokuma K, Zakrzewski VG, Voth GA, Salvador P, Dannenberg JJ, Dapprich S, Daniels AD, Farkas O, Foresman JB, Ortiz JV, Cioslowski J, Fox D J (2009) Gaussian Inc.

13. Boys F, Bemardi F (1970) The calculation of small molecular interactions by the differences of separate total energies. Some procedures with reduced errors, Mol. Phys 19:553-566. https://doi.org/10.1080/00268977000101561

14. Lu T, Chen F (2012) Multiwfn: a multifunctional wavefunction analyser J. Comput. Chem 33:580-592. https://doi.org/10.1002/jcc.22885

15. Bader RFW (1985) Atoms in molecules. Acc. Chem. Res. 18:9-15 https://doi.org/10.1021/ar00109a003. 
16. Bader RFW, Streitwieser A, Neuhaus A, Laidig KE, Speers P (1996) Electron Delocalization and the Fermi Hole. J Am Chem Soc 118:4959- 4965. https://doi.org/10.1021/ja953563x

17. Bader, RFW (1990) Atoms in Molecule A quantum Theory. Oxford Univ press, Oxford

18. Becke AD, Edgecombe KE (1990) A simple measure of electron localization in atomic and molecular systems. J Chem Phys 92 5397. https://doi.org/10.1063/1.458517

19. Lu T, Chen F (2012) Quantitative analysis of molecular surface based on improved Marching Tetrahedra algorithm J Mol Graph Model 38:314-323. doi: 10.1016/j.jmgm.2012.07.004. Epub 2012 Jul 27.

20. Lu T, Chen FA (2012) Multifunctional wavefunction analyzer J Comp Chem 33:580-592. https://doi.org/10.1002/jcc.22885

21. Savin A, Flad HJ, Flad J, Preuss H, von Schnering HJ. (1992) On the bonding in Carbosilanes. Angew Chem Int EdEngl. 31(2):185-187. https://doi.org/10.1002/anie.199201851

22. Becke AD (2000) Chemical content of the kinetic energy density J Mol Struct (THEOCHEM) 527: 51-56

23. Khalili Z, Darvish Ganji M, Mehdizadeh M (2018) Fluorouracil functionalized Pt-doped carbon nanotube as drug delivery nanocarrier for anticarcinogenic drug a B3LYP-D3 study J Nanoanalysis 5(3):202-209. doi: 10.22034/jna.2018.543692

24. Shahabi M, Raissi H (2017b) Screening of the structural, topological, and electronic properties of the functionalized graphene nanosheets as potential Tegafur anticancer drug carriers using DFT method. J Biomol Struct Dyn 36(10): 2517-2529. doi: 10.1080/07391102.2017.1360209

25. Dastani N, Arab A, Raissi H (2020) Adsorption of Ampyra anticancer drug on the graphene and functionalized graphene as template materials with high efficient carrier Adsorption 26:879-893. https://doi.org/10.1007/s10450-019-00142-1

26. Steiner E, Fowler P W (2001) Patterns of Ring Currents in Conjugated Molecules: a few-electron model based on orbital contributions. The Journal of Physical Chemistry A 105(41): 9553-9562. doi: 10.1021/jp011955m

27. Steiner E, Fowler PW, Havenith RWA (2002) current densities of localized and delocalized electrons in molecules, the journal of physical chemistry A 106(30): 7048-7056. doi: 10.1021/jp020819u

28. Frost A, Musulin, B (1953) A mnemonic device for molecular orbital energies J. Cheme Physic 21: 572. https://doi.org/10.1063/1.1698970

29. Jalali Sarvestani M, Ahmadi R (2017) Investigating the Complexation of a Recently Synthesized Phenothiazine with Different Metals by Density Functional Theory. Int J New Chem 4(4):101-110. doi:10.22034/IJNC.2017.30984

30. Jalali Sarvestani M, Majedi S (2020) A DFT study on the interaction of alprazolam with fullerene (C20) J Chem Lett. 1(1):32-38. Doi: 10.22034/JCHEMLETT.2020.108111

31. Hamzehloueian MA (2017) Density functional theory study on the reaction mechanism of hydrazones with a-oxo-ketenes Comparison between stepwise 1,3-dipolar cycloaddition and DielseAlder pathways C R Chim 20(5):508-519. doi:10.1016/j.crci.2016.09.002

32. Safdari F, Raissi H, Shahabi M, Zaboli M (2017) DFT calculations and molecular dynamics simulation study on the adsorption of 5-fuorouracil anticancer drug on graphene oxide nanosheet as a drug delivery vehicle J Inorg Organomet Polym Mater 27:805-817. https://doi.org/10.1007/s10904-017-0525-9 
33. Kanis DR, Ratner MA, Marks TJ (1994) Design and construction of molecular assemblies with large second-order optical nonlinearities Quantum chemical aspects Chem Rev 94(1):195-24. https://doi.org/10.1021/cr00025a007

34. Thanthiriwatte KS, De Silva KMN (2002) Non-linear optical properties of novel fluorenyl derivatives-ab initio quantum chemical calculations J Mol Struct THEOCHEM. 617:169-175. doi:10.1016/S01661280(02)00419-0

35. Kleinman DA (1962) Nonlinear Dielectric Polarization in Optical Media Phys Rev. 126(6), 1977-1979. http://dx.doi.org/10.1103/PhysRev.126.1977

36. Shokuhi Rad A, Aghaeib SM, Aalia E, Peyravic M (2017) Study on the electronic structure of Cr- and Nidoped fullerenes upon adsorption of adenine a comprehensive DFT calculation, Diam Relat Mater 77:116121. doi:10.1016/j.saa.2020.119082

37. Arulmozhi S, Madhavan J (2015) Molecular structure, First-order hyperpolarizability and HOMO-LUMO studies of L- Histidinium Dinitrate International Conference on Materials Science and Technology 73:012035. https://doi.org/10.1088/1757-899X/73/1/012035

38. Yang Y, Ostadhosseini NA (2021) Theoretical investigation on the mercaptopurine drug interaction with boron nitride nanocage solvent and density functional effect Phys E Low-dimens Syst Nanostruct 125:114337. doi:10.1016/j.physe.2020.114337 